\title{
« Géographie de l'exil » de Juan Goytisolo
}

Sur les traces génétiques et intertextuelles de Paysages après la bataille

\section{Bénédicte Vauthier}

\section{(2) OpenEdition}

Journals

Édition électronique

URL : http://journals.openedition.org/genesis/1080

DOI : $10.4000 /$ genesis. 1080

ISSN : 2268-1590

Éditeur :

Presses universitaires de Paris Sorbonne (PUPS), Société internationale de génétique artistique littéraire et scientifique (SIGALES)

\section{Édition imprimée}

Date de publication : 15 novembre 2012

Pagination : 221-234

ISBN : 978-2-84050-869-4

ISSN : 1167-5101

\section{Référence électronique}

Bénédicte Vauthier, « « Géographie de l'exil » de Juan Goytisolo », Genesis [En ligne], 35 | 2012, mis en ligne le 15 novembre 2014, consulté le 19 juin 2020. URL : http://journals.openedition.org/genesis/ 1080 ; DOI : https://doi.org/10.4000/genesis. 1080 


\title{
"Géographie de l'exil 》 de Juan Goytisolo Sur les traces génétiques et intertextuelles de Paysages après la bataille
}

\author{
Bénédicte Vauthier
}

$\mathrm{P}$ ublié en espagnol en 1982 et traduit trois ans plus tard en français, Paysages après la bataille (1985¹), roman de maturité de l'écrivain espagnol Juan Goytisolo (1931), se présente dès la quatrième de couverture de la traduction comme « un puzzle dont les morceaux se mettent en place au fur et à mesure du déroulement du roman ». C'est dans les méandres de cette construction mystérieuse, qui, en réalité, tient plus encore du casse-tête ${ }^{2}$ que du puzzle, que je souhaite me plonger ici.

En rupture claire avec les codes du réalisme - linéarité de la diégèse, unicité du sujet, caractère organique de l'œuvre -, Paysages après la bataille s'écrit ouvertement sous le signe d'une esthétique du fragment ${ }^{3}$, qui, de l'aveu de l'auteur, s'enracine dans son expérience de villes protéiformes, plurielles, bigarrées comme New York, Berlin ou plus encore Paris, toile de fond du roman ( $\mathrm{PaB}, \mathrm{p}$. 15).

En cherchant à emboîter le pas du protagoniste anonyme, alter ego autofictionnel de l'auteur dont les déambulations recoupent celles du flâneur de Walter Benjamin, nous sommes amenés à nous perdre « dans le Paris des trajets qui bifurquent » (PaB, p. 104).

Pour retrouver le nord, tout nous invite à multiplier les pistes de lecture des soixante-dix-sept fragments de ce roman mosaïque, dont la structure spatio-temporelle déjà éclatée est encore diffractée sous l'effet de trois axes narratifs qui renvoient à trois facettes de la personnalité du protagoniste (sexualité, engagement politique et écrivainchroniqueur-copiste).

Jeu de cartes qui semblent pouvoir être battues à l'infini ; livre semblable au métro parisien, dont l'espace est «vaste, riche en possibilités : ramifications, croisements, passages, voies à sens unique, paraboles, demi-tours, ellipses, déviations » (PaB, p. 104-105), Paysages après la bataille est aussi un livre circulaire qui invite « à une lecture arabe, de droite à gauche, de la prétendue fin au faux début, en partant de la genèse de son écriture pour remonter à la monstrueuse hécatombe ${ }^{4}$ ».

Mais qu'en est-il au juste de cette genèse de l'écriture? N'est-elle que désordre, comme peut le laisser entendre cette présentation?

Lisons tout d'abord ce que dit l'auteur à son propos dans le prologue au troisième volume de ses CEuvres complètes, dont il assure l'édition depuis 2005, livrant au généticien de nombreuses indications sur ses habitudes de travail.

La composition de Paysages après la bataille ne répondait à aucun plan. J'ai rédigé une douzaine de textes indépendants les uns des autres et à partir de leur relecture j'ai capté leurs affinités secrètes, j'ai cherché la manière de mettre en relation leur archipel ou d'intégrer celui-ci dans un puzzle dont j'ignorais la structure. Ce travail à tâtons, non à l'aveuglette, prenait peu à peu consistance et je me souviens de l'après-midi où, alors que je me promenais seul dans le jardin de Torrentbó,

1. Les renvois à la traduction française de Paysages après la bataille seront signalés dans le corps du texte par l'abréviation $\mathrm{PaB}$, suivie de la page citée.

2. C'est l'expression qui est utilisée sur la quatrième de couverture de l'édition originale pour qualifier le roman.

3. Cette fragmentation du roman est caractéristique d'une bonne partie de l'œuvre romanesque goytisoléenne des années quatre-vingt. Outre Paysages après la bataille, je pense à Makbara et aux deux volumes autobiographiques : Chasse gardée et Les Royaumes déchirés. On la retrouve encore dans des textes plus tardifs, comme La Saga de los Marx (1993). Cette esthétique du fragment peut être mise en rapport, d'une part, avec la crise du sujet qu'expérimente l'auteur à la fin des années soixantedix, années d'écriture de la trilogie de la rupture ; d'autre part, avec l'œuvre de Walter Benjamin, que Goytisolo découvre et cite abondamment au cours des années quatre-vingt.

4. Je traduis ici quelques lignes extraites de la quatrième de couverture de la première édition hispanophone du roman (Mexico, Montesinos, 1982). 
j'ai clairement perçu ce que devait être le livre et comment assembler les pièces de ce casse-tête (Goytisolo, 2006, III, p. 39 [je traduis]).

Et voyons maintenant si le dossier génétique est à même de nous mettre sur la piste de cette douzaine de textes.

\section{Dossier génétique}

Avant de passer à l'analyse du dossier génétique de Paysages après la bataille, commençons par rappeler que les manuscrits de travail de Juan Goytisolo sont aujourd'hui dispersés entre l'Université de Boston (œuvres de jeunesse et de maturité, de 1955 à 1975), la bibliothèque municipale d'Almería (années 1980-1990), Marrakech (Makbara, 1980) et le domicile de l'auteur (œuvres tardives, c'est-àdire de 1990 à nos jours) ${ }^{5}$. Les brouillons de Paysages après la bataille sont ainsi conservés à Almería, à l'exception, toutefois, de l'édition du roman (1987) dans laquelle Juan Goytisolo a apporté des corrections qui ont été intégrées dans ses œuvres complètes en cours de publication (2006), qui se trouve elle à son domicile6.

Le dossier génétique de Paysages après la bataille est composé de deux sous-ensembles : le premier compte quatre cent quatre-vingt-trois pages et forme ce que la responsable de la bibliothèque d'Almería m'avait présenté comme « le manuscrit » du roman ${ }^{7}$. Les pages ont été classées et foliotées par une main étrangère dans le coin supérieur droit en tenant compte de l'ordre des chapitres de la seconde édition publiée à Barcelone (1985), comme le révèle une note manuscrite anonyme jointe aux brouillons. Ce classement hypothèque lourdement toute tentative de reconstruction de la genèse initiale des fragments. Le second sous-ensemble, également folioté en chiffres romains, est constitué de trois cent cinquante-neuf pages, laissées dans le plus grand désordre. Il s'agit des brouillons de dix-huit des vingt et une séquences non reprises dans la première chemise ou, pour le dire autrement, l'autre moitié du « manuscrit ${ }^{8}$ ». Au total, il ne manque que les brouillons de trois des soixante-dix-sept chapitres ou fragments constitutifs du roman ${ }^{9}$. Leur absence peut s'expliquer par le fait qu'il s'agit de trois fragments intégrés dans le roman après avoir fait l'objet d'une première publication sous forme de « Tribune » dans la presse natio- nale. Nous avons là une première trace génétique de l'existence préalable de documents autotextuels 10 qui sont entrés dans la composition du roman.

À quelques exceptions près, les brouillons repris dans les deux chemises (rassemblées aujourd'hui dans un portefeuille cartonné moucheté) (fig. 1) se présentent de la même manière : Juan Goytisolo écrit toujours au Bic bleu, soit sur des feuilles A4 $(21 \times 29,7 \mathrm{~cm})$ qu'il plie en deux pour former de petits cahiers ou feuillets doubles de quatre pages, soit sur des feuilles de bloc de format A5 $(14,7 \times 21 \mathrm{~cm})$. Il remplit entièrement la page d'une petite écriture régulière et droite, sans laisser de marges latérales. En général, il commence toutefois le premier recto d'une nouvelle séquence en laissant une marge supérieure de trois centimè-

5. En décembre 2011, Juan Goytisolo a signé un accord concernant ses archives avec le ministère de la Culture espagnol, et les documents qu'il conserve aujourd'hui encore à son domicile iront aux Archives générales de l'administration, à Alcalá de Henares, à une trentaine de kilomètres de Madrid.

6. Le roman a fait l'objet de plusieurs éditions, qui ont été prises en considération. À l'édition princeps de 1982, font suite les éditions catalanes de 1985 (Llibres del Mall) et 1987 (Galaxia Gutenberg/Círculo de Lectores), illustrées par le dessinateur Eduardo Arroyo, et une édition de poche (Austral, 1990), qui suit l'édition de 1987. Depuis 2005, Juan Goytisolo s'est lancé dans une édition de ses œuvres complètes. Celles-ci sont établies par Antonní Munné sur la base des dernières éditions revues et corrigées par l'auteur. En ce qui concerne Paisajes después de la batalla, Juan Goytisolo a introduit une série de corrections dans son exemplaire de l'édition de 1987. Celui-ci est donc venu grossir, d'une manière tardive et inattendue, le dossier génétique de l'œuvre.

7. Pour une présentation exhaustive du dossier, je renvoie aux préliminaires et annexes génétiques de mon édition de Paisajes después de la batalla (Salamanca, Ediciones Universidad de Salamanca, 2012b).

8. À partir de maintenant, je ferai référence à ces deux chemises en les désignant par MSI et MSII.

9. Dans la version de Paysages après la bataille reprise dans les Euvres complètes (2006), l'auteur a supprimé le chapitre « Pour elle », considérant que son caractère par trop autobiographique détonnait dans le reste du récit (2006). Le roman ne compte donc plus aujourd'hui que soixante-seize et non soixante-dix-sept chapitres. Pour l'analyse génétique des brouillons de ce fragment et les effets de sens de la suppression, voir Vauthier, 2012b. 10. Selon la préfiguration en ligne du Dictionnaire de critique génétique de l'ITEM (version du 21 décembre 2010), par autotextualité, on comprend « l'ensemble des relations d'un texte avec lui-même. Dans le cadre de la genèse, ces relations peuvent s'appliquer aux phénomènes de réécriture ». En ce qui me concerne, je n'englobe sous ce terme que des faits d'autocitation cachée ou de déplacements entre œuvres propres (<www.item.ens.fr/index.php?id=577462>). 
tres, qui peut ensuite être occupée par un titre. Généralement ajouté de manière postérieure à l'écriture de la séquence, comme le révèle le grand nombre de premiers brouillons dépourvus de titre, celui-ci est alors toujours centré, écrit en caractères ronds et souligné dans une première version, puis centré et en petites capitales dans une mise au net qui pourrait être considérée comme définitive, si rares n'étaient, en fin de compte, les mises au net qui correspondent réellement au texte publié. Cette remarque nous permet de préciser qu'il existe de deux à sept versions d'une même séquence - la moyenne étant de trois ou quatre -, auxquelles s'ajoutent de possibles couches d'écriture observables au sein d'un même brouillon, ce qui est particulièrement vrai dans le premier état d'un fragment ${ }^{11}$. Le grand nombre de versions d'une même séquence est également lié à la méthode de travail de l'auteur : écriture et travail de copie, puis de recopiage se font toujours à la main.

J'écris encore toujours à la main et pour faciliter le travail autrefois des dactylographes et maintenant des informaticiens, j'essaie de le faire d'une calligraphie très claire. Je n'ai jamais appris à taper sur une Remington ou sur une Olivetti, ni même ne m'y suis essayé, car je suis convaincu de ma maladresse innée eu égard à la technique et aux choses pratiques. À l'époque des ordinateurs et des courriers électroniques, je continue, à l'encontre de toute notion de progrès, mon travail artisanal manuscrit. Autrefois je le faisais avec un stylo de marque que j'ai abandonné, déjà à Paris, au profit du stylo à bille qui est plus commode et plus maniable. Celui-ci et la bande correctrice destinée à couvrir les ratures afin de rendre la page corrigée à la main plus lisible sont les seules avancées technologiques que j'aie pu assimiler de manière bénéfique au cours de ma vie (Goytisolo, 2005, I, p. 18-19 [je traduis]).

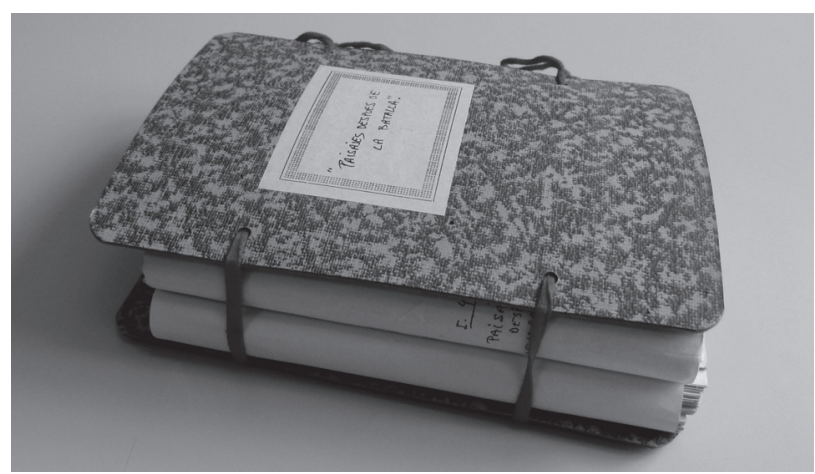

Fig. 1 : Dossier génétique (@ Elías Palmero, Fonds Goytisolo)
Outre ces brouillons autographes et deux pages dactylographiées, le dossier compte également quelques coupures de presse, en français, en espagnol et en anglais, qui forment une sorte de « préécrit », c'est-à-dire « cette partie visible de l'iceberg des discours de référence historico-culturelle, celle qui nous est transmise dans le dossier génétique comme ayant participé de l'élaboration textuelle » (Grésillon, 1994, p. 172). Cette notion nous semble encore particulièrement intéressante aujourd'hui, car elle permet de distinguer « strictement les documents qui ont laissé des traces écrites dans le dossier génétique d'une œuvre » (ibid.) du cas plus large de l'intertextualité, déclarée ou implicite ${ }^{12}$.

\section{Les feuilles de notes}

Parmi les trois cent cinquante-neuf pages de brouillon de la seconde chemise, nous trouvons également trois feuilles A4 pliées en deux pour former des feuillets doubles, dont l'aspect graphique est atypique par rapport au gros des brouillons décrits précédemment. Il s'agit respectivement des pages MSII 153-156, 201-204 et 205-208. À la différence des séquences narratives linéarisées, on peut y voir présentée de manière schématique une série de thèmes ou d'idées qui ont été développés dans des séquences du roman, témoignage ou trace vraisemblable de la première étape de construction à laquelle faisait allusion Juan Goytisolo.

Comme on peut l'observer sur la page MSII 20113 (fig. 2), ces idées sont parfois circonscrites par des encadrements, dont la plupart ont été hachurés de manière

11. Dans le cadre d'un travail de collaboration avec Malte Rehbein (Zentrum für digitale Edition, Universität Würzburg), une édition génétique numérique de quelques séquences représentatives du travail d'écriture de l'écrivain est en train d'être mise en place. La plateforme numérique vise à ressaisir la dynamique du processus d'écriture et donc la temporalité de l'écriture, que le manuscrit a figée.

12. La notion n'a toutefois pas rencontré beaucoup d'écho parmi les généticiens, peut-être parce qu'elle recoupe en partie celles d'exogenèse et d'endogenèse, largement acceptées (voir de Biasi, 1998).

13. Tous droits réservés. Que soient remerciés ici Juan Goytisolo, qui m’a généreusement autorisée à consulter et à reproduire ses brouillons dans le cadre de mon travail d'édition, et María Carmen Amate, responsable de la bibliothèque de la « Diputación provincial d'Almería », qui a aimablement mis ceux-ci à ma disposition. 


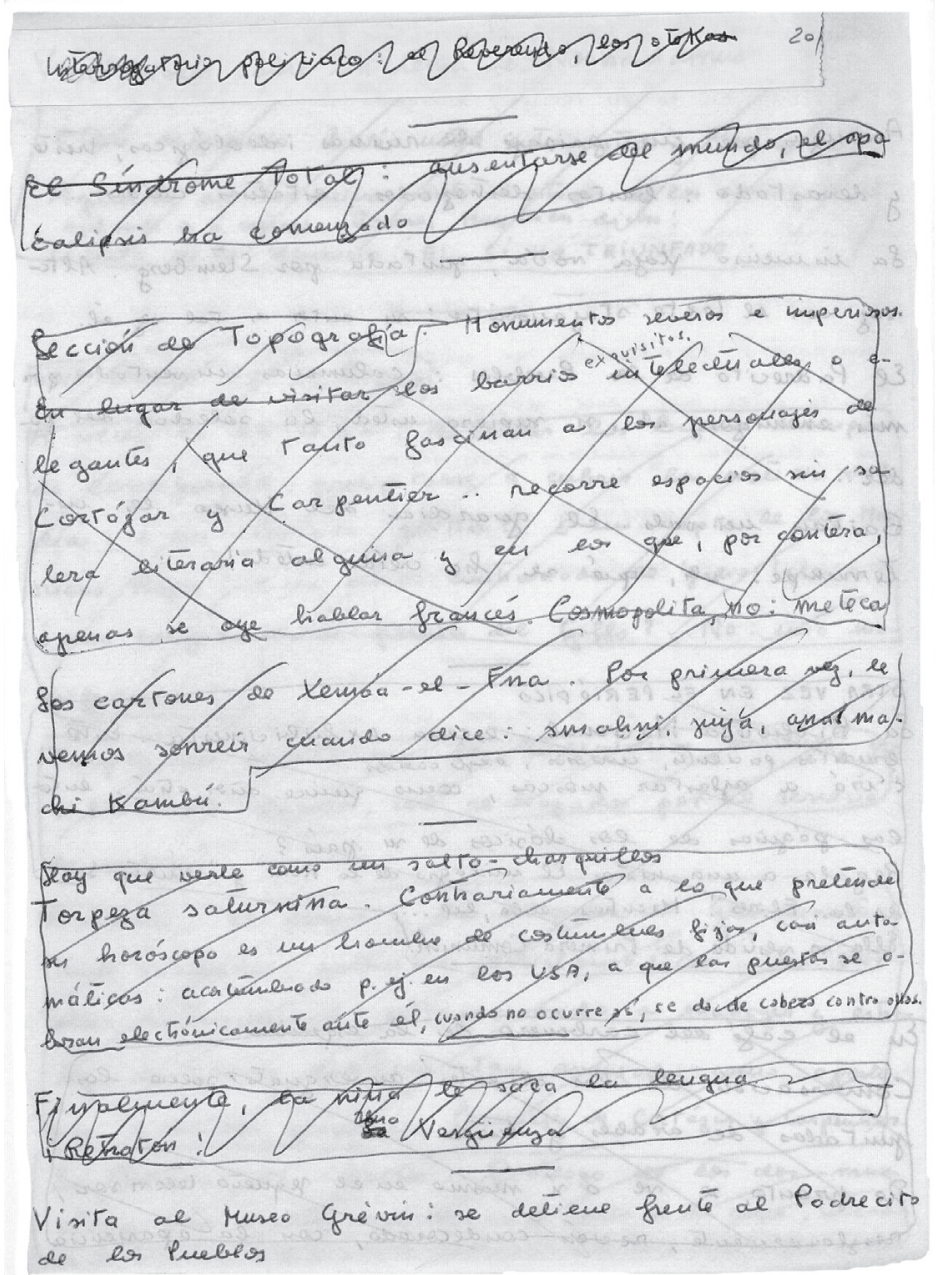

Fig. 2 : MSII 201 (@ Fonds Goytisolo)

oblique ou par une croix de Saint-André, comme s'il s'agissait d'une liste de tâches à effectuer, éliminées une fois accomplies ${ }^{14}$. De plus, certains thèmes sont repris d'une page à l'autre - des pages MSII 205-207 à MSII 201-204, puis à nouveau sur les pages MSII 153-155, les pages 156 et 208 étant restées vierges -, ce qui laisse penser que nous nous trouvons là aussi face à trois états différents. Dans la première version, nous aurions une dizaine de séquences ou chapitres potentiels, alors que dans les deux états suivants, nous en aurions une quinzaine, dont certains qui se répètent.

Bien plus encore, on verra que la page MSII 153 révèle la possible existence d'un titre devenu excipit, « lieu stratégique du texte imprimé », comme l'a montré Debray Genette pour Un cœur simple de Gustave Flaubert15, luimême écarté par la suite pour une nouvelle fin romanesque trouvée tardivement. Finalement, ces déplacements et ces réécritures dont le texte publié n'a pas gardé la trace semblent avoir entraîné dans leur sillage l'effacement d'un dédicataire (Leïla Sebbar) et d'une référence intertextuelle (la revue de l'immigration Sans Frontières) qui, d'explicites dans un état avancé de la rédaction, sont devenus implicites dans la «première version définitive » de l'œuvre.

Ceci étant dit, il ne peut être question de retranscrire ici ces dix pages de notes ${ }^{16}$. Je vais me contenter d'in-

14. Ces ratures pourraient être rapprochées des « ratures d'utilisation» dont parle Pierre-Marc de Biasi pour désigner « les éléments préparatoires qui ont déjà été utilisés et sur lesquels il n'y a plus lieu de revenir » (de Biasi, 2000, p. 57).

15. Raymonde Debray Genette utilise le terme excipit (en lieu et place d'explicit) pour désigner le(s) dernier(s) paragraphe(s) d'une œuvre. Ce « terme a le mérite de nommer avec exactitude l'inverse de l'incipit » et ce même si les fonctions des deux segments ne sont pas ensuite simplement renversables (1988, p. 85).

16. Ces dix pages de notes sont reproduites en fac-similés et en transcriptions linéarisées dans les préliminaires de mon édition (Vauthier, 2012b). 
diquer brièvement à quel chapitre elles appartiennent en me basant sur des idées-clés ou des syntagmes qui sont présents tant dans ces notes synthétiques que dans le roman. Je précise d'entrée de jeu que c'est moi qui indique ici et la situation du chapitre (ou fragment) dans le roman (chiffre arabe sur la gauche) et le titre qu'il portera.

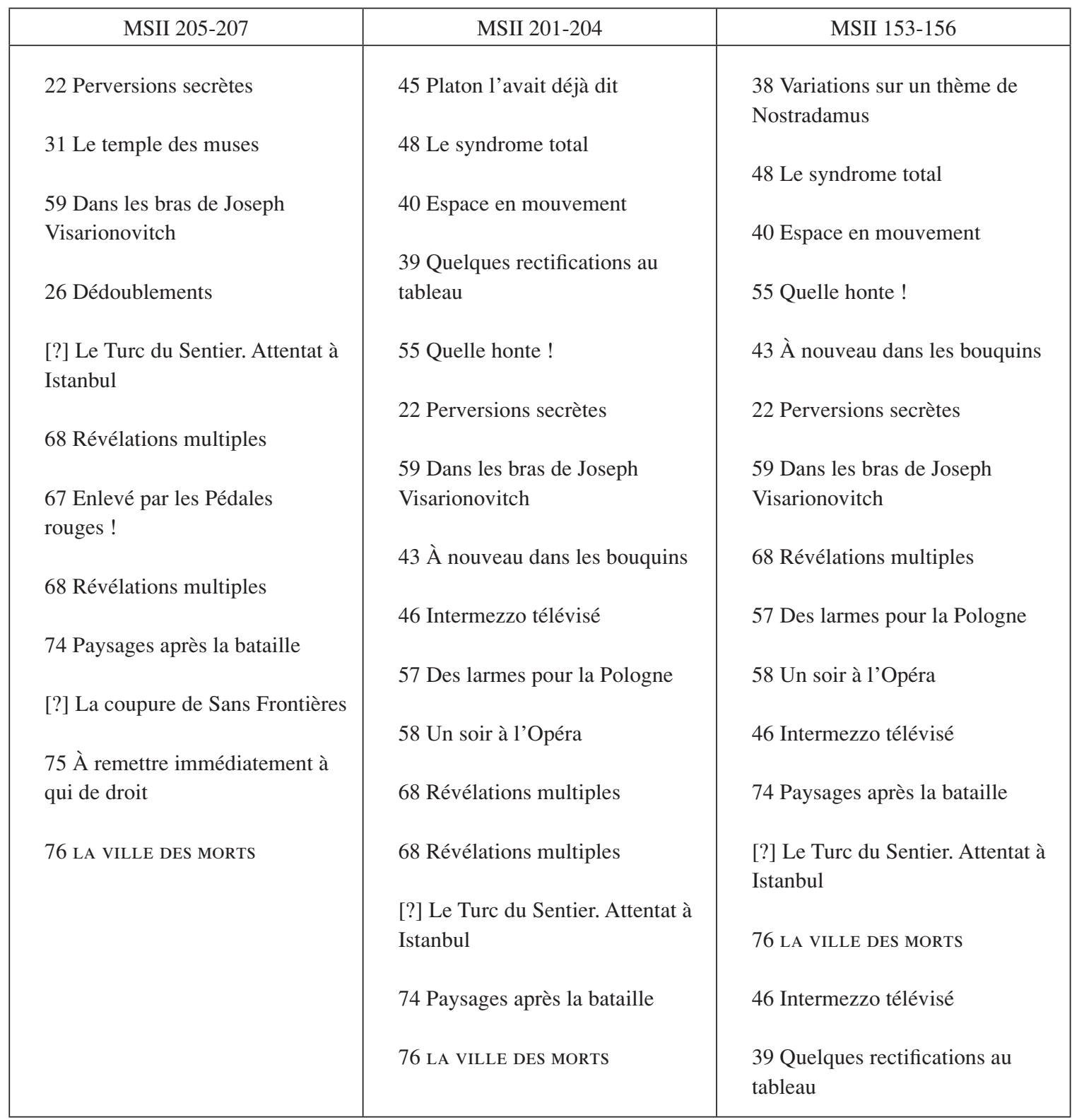




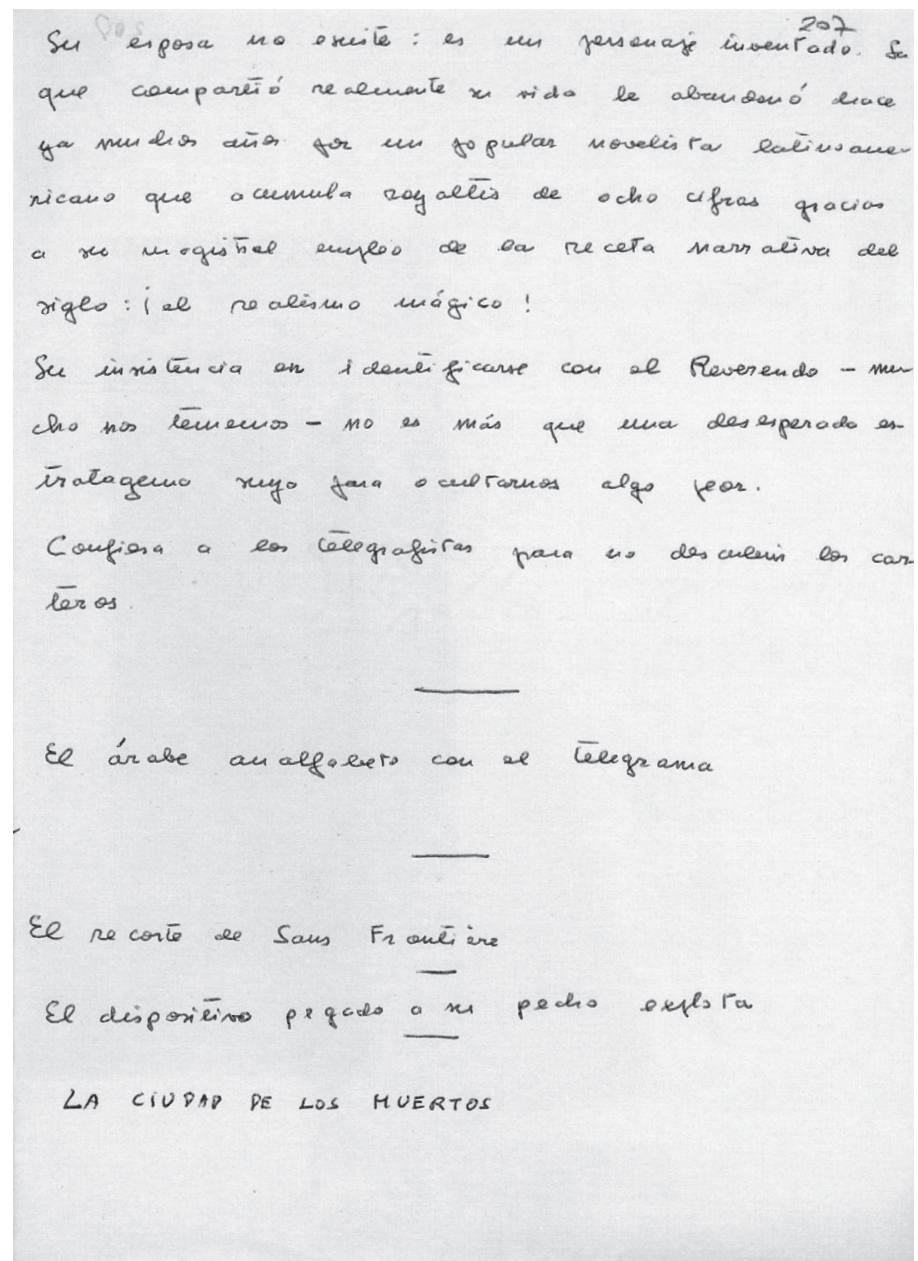

Fig. 3 : MSII 207 (@ Fonds Goytisolo)

Une comparaison des entrées de ce tableau récapitulatif, notamment les trois premières de la deuxième colonne, qui correspond aux ratures du début de la page MSII 201, que je transcris ici, illustre bien le caractère synthétique ou synoptique de ces feuilles atypiques.

Interrogatorio policiaco: el Reverendo, los otekas [Interrogatoire policier : le Révérend, les otèques]

El síndrome total: ausentarse del mundo, el apocalípsis ha comenzado [Le syndrome total : s'absenter du monde, l'apocalypse à commencé]

Lección de topografía: Monumentos severos e imperiosos. En lugar de visitar los barrios exquisitos intelectuales o elegantes, que tanto fascinan a los personajes de Cortázar y Carpentier... recorre espacios sin sa... [Leçon de topographie: Monuments sévères et impérieux. Au lieu de visiter les quartiers exquis, intellectuels ou élégants qui fascinent tellement les personnages de Cortázar et de Carpentier... il parcourt des espaces sans...]
De ces trois entrées, seule la deuxième reprend ici le « titre littéral » d'un chapitre du roman. Dans les deux autres cas, ce sont les thèmes abordés (interrogatoire policier) ou les mots-clés (Leçon de topographie, Carpentier, Cortázar) qui m'ont permis leur localisation dans le roman.

$\mathrm{Si}$ on compare les trois étapes rédactionnelles, on voit que six séquences (ou chapitres) de la première version se répètent dans les deux autres. Il s'agit respectivement des séquences : 22, 59, 68, « Le Turc du Sentier», 74 et 76. Outre «Révélations multiples », chapitre décisif, car le narrauteur 17 y livre des clés pour une interprétation idoine du roman, dans les trois versions on retrouve deux des trois séquences finales (74/75 et 76), auxquelles pourrait peutêtre s'ajouter le fragment [73], «La prédiction du mara-

17. À la suite d'Elsa Dehennin, par « narrauteur » ou «narrateur communicateur », je me réfère à une instance narrative plus proche de l'auteur que du simple narrateur à la première ou à la troisième personne. Cette instance correspond grosso modo à l'auteur implicite (the implied author) dans la tradition anglo-saxonne (Dehennin, 1996, p. 141). 


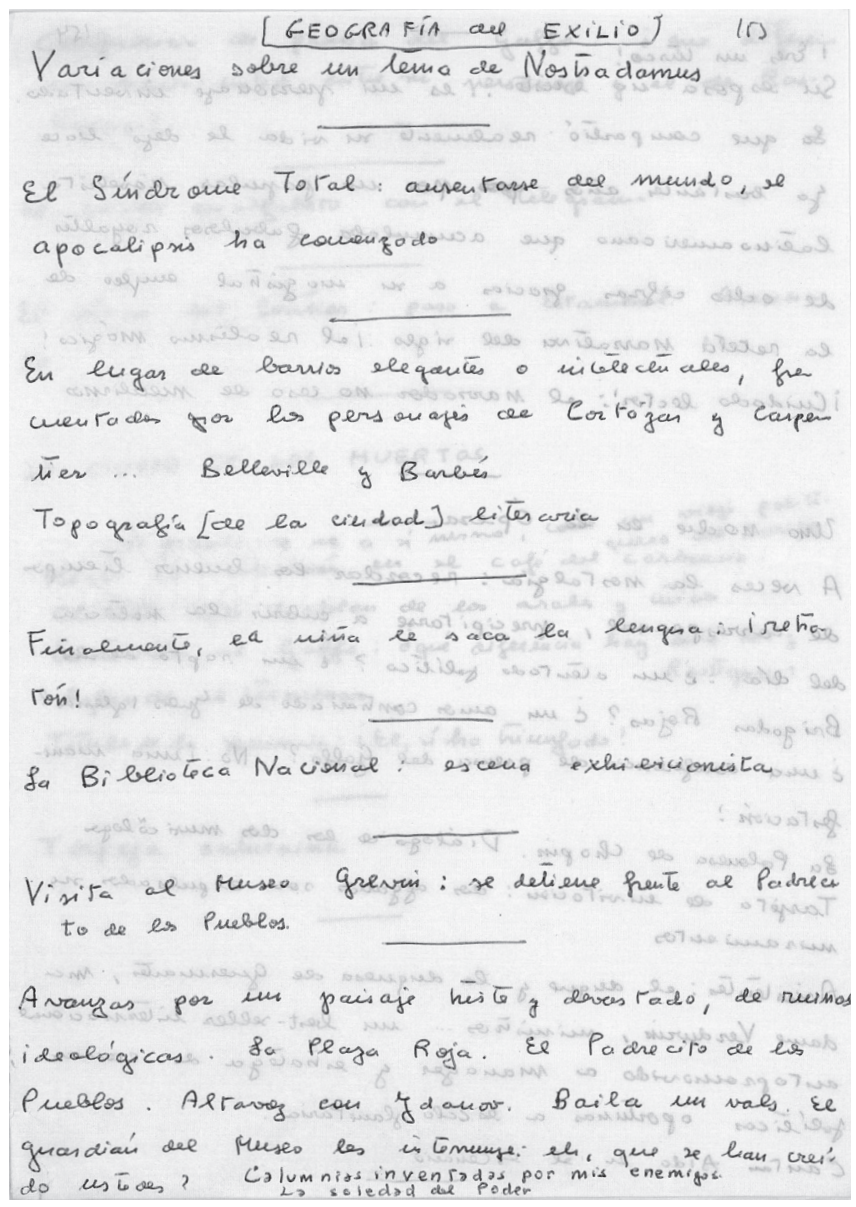

Fig. 4 : MSII 153 (@ Fonds Goytisolo)

bout », qui fait état de l'immolation d'un individu de couleur « dans le vestibule de la gare du Nord», ce qui pourrait renvoyer au « Turc du Sentier. Attentat à Istanbul », idées qui n'apparaissent pas telles quelles dans le roman. La séquence [22], « Perversions secrètes », qui a lieu au musée Grévin, est en relation étroite avec les séquences 57 , 58 et 59. Dans ces quatre fragments, on voit apparaître « le Petit Père des Peuples », héros détrôné qui permet à l'auteur de régler ses comptes avec le communisme ou de tempérer son enthousiasme juvénile pour la gauche.

Dans la première version, nous avons également une référence unique à trois chapitres $(31,26$ et 67) qui, bien qu'absents des deux autres étapes rédactionnelles, font partie du roman. Il n'en va pas de même de la mention d'une coupure de Sans Frontières («El recorte de Sans Frontières », MSII 207, fig. 3), ce qui rend la référence particulièrement suggestive, car cette coupure de presse ne figure pas dans le dossier génétique, pas plus que son titre ne figure dans le roman, alors que les reportages de cette
Revue sur l' immigration et le Tiers-Monde - l'un des axes porteurs du roman - pourraient être inscrits à son origine, comme nous ne tarderons plus à le voir.

Exception faite de la séquence «Platon l'avait déjà dit » (MS II 201), toutes les séquences mentionnées dans la deuxième version le sont dans la troisième, et l'ordre de leur apparition est à peine altéré.

Dans la troisième version (MSII 153, fig. 4), nous avons une référence isolée aux «Variations sur un thème de Nostradamus », titre original de l'une des séquences du roman dont le contenu reproduit partiellement le dernier paragraphe («Remítase a los astros ») d'une seconde « Tribune » de l'auteur, « Telediario 1984 », publiée dans El País en décembre 1981, avant d'être démembrée et intégrée dans le roman (Vauthier, 2012a, 2012b). À la différence du premier élément autotextuel mentionné ci-dessus, les brouillons de cette seconde tribune se trouvent dans le dossier génétique de Paysages après la 


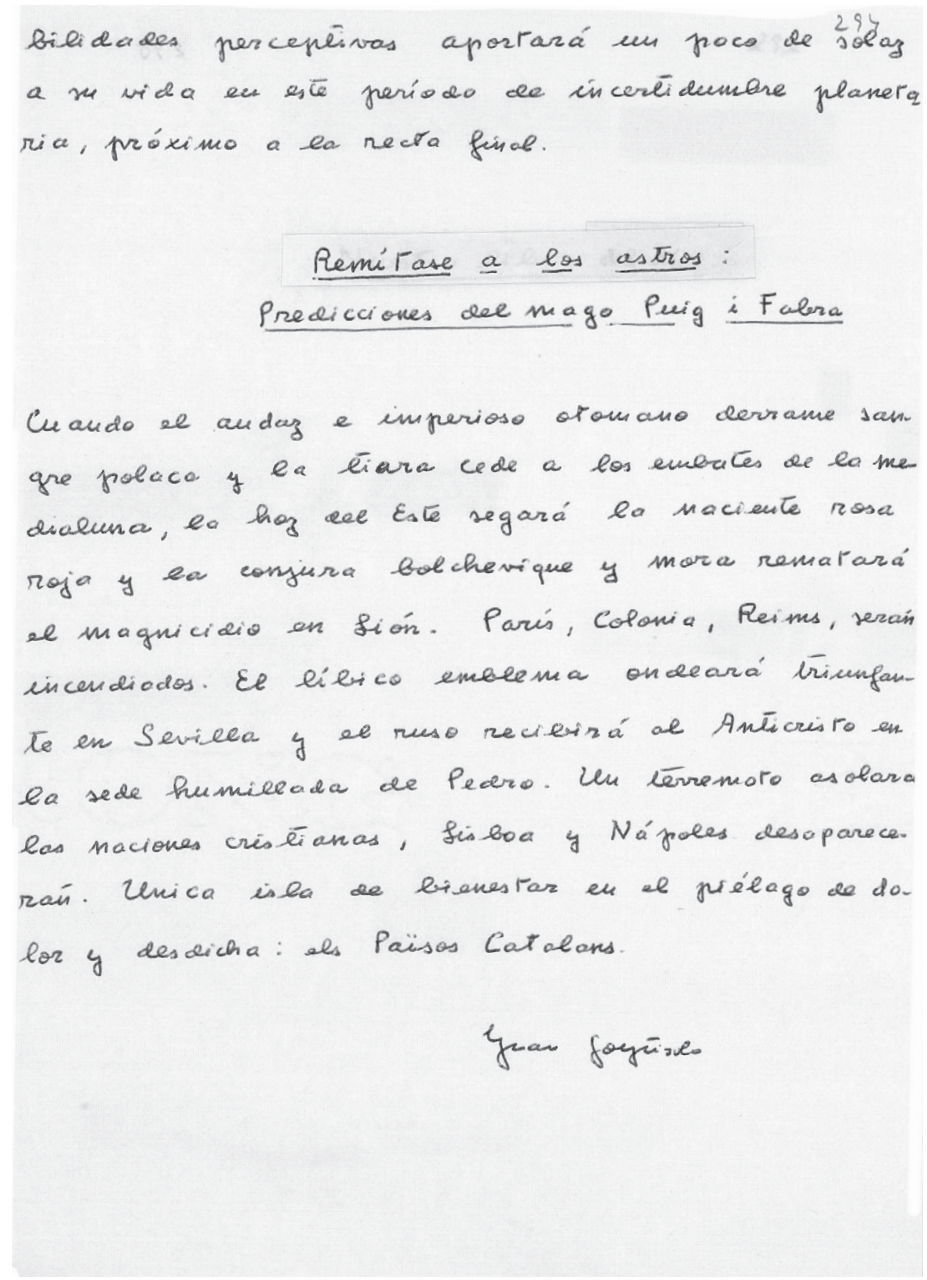

Fig. 5 : MSII 294 (@ Fonds Goytisolo)

bataille, qui comprend aussi la mise au net de l'article avec la signature de l'auteur et le fragment final de l'article de El País (MSII 294, fig. 5).

Ce nouvel élément autotextuel, qui fait également partie du préécrit, me permet d'attirer l'attention sur la première caractéristique de bon nombre des séquences reprises dans ces feuilles synthétiques, à savoir que plusieurs d'entre elles ont partie liée avec les coupures de presse conservées dans le dossier génétique ${ }^{18}$ ou avec l'intertextualité qui sous-tend le roman. L'ensemble de ce matériel renvoie à deux dimensions clés de la personnalité du héros : celle de collectionneur, d'une part, celle de copiste, d'autre part, deux facettes en lien avec l'intertextualité flaubertienne « la filiation » (Goytisolo, 2000, p. 37) - sous-jacente à la forme et au contenu de l'ouvrage qui s'ouvre sur une épigraphe tirée de Bouvard et Pécuchet. À cette première référence intertextuelle convoquée explicitement au seuil du livre ${ }^{19}$, tout comme l'est également l'œuvre photogra- phique et épistolaire de Lewis Carroll20, il faut ajouter les écrits de Walter Benjamin et de Karl Kraus, dont les noms apparaissent dans le paratexte auctorial, contemporain ou postérieur à l'écriture du roman. Citons, pour exemple, cette mention tardive de Walter Benjamin : « La plus grande dette de ce livre, exception faite de l'hommage à Flaubert, est celle que j'ai contractée envers la vision déstabilisatrice de Baudelaire et de la lecture qu'en fait Walter

18. Concrètement, il s'agit des chapitres ou séquences $22,31,38,39,40$ et 43 (voir Vauthier, 2012b).

19. «Ils mettaient en doute la probité des hommes, la chasteté des femmes, l'intelligence du gouvernement, le bon sens du peuple, enfin sapaient les bases. »

20. Sur la quatrième de couverture de l'édition princeps, les par trop pressants fantasmes sexuels du protagoniste étaient mis en relation avec les fantasmes sexuels du créateur d'Alice au pays des merveilles, attiré par les jeunes filles, et la photo la plus célèbre de la jeune inspiratrice de chaire et d'os du récit (Alicia Liddell) ornait la première de couverture avant de servir de modèle à une ekphrasis (voir Vauthier, 2012b). 
Benjamin, comme je l'ai expliqué dans "Pourquoi j'ai décidé de vivre à Paris" et "Paris, capitale du Xxie siècle" » (2006, III, p. 39). Tout ce matériel - préécrit et intertextualité - renvoie enfin à une dimension constitutive de l'œuvre de Juan Goytisolo, qui se plaît à reconnaître l'importance du greffon littéraire sur l'arbre de la littérature (Goytisolo, 2009, VI, p. 1056).

\section{Paysages après la bataille, fuyante « Géographie de l'exil »}

Cette relation initiale du roman avec d'autres textes, qui sont détournés de leur fonction et de leurs supports originaux par le biais de la traduction, de la stylisation, de la parodie ou du clin d'œil intertextuel, rend d'autant plus significative, comme je le disais plus haut, l'absence d'une possible coupure de presse tirée de la revue Sans Frontières explicitement nommée sur la page MSII 207. Et l'absence devient plus parlante encore à la lumière de Géographie de l'exil, possible titre inscrit en haut de la page MSII 153, dans un petit encadrement semblable à celui que nous imitons ici (fig. 4).

Or ce titre potentiel, amené à disparaître en tant que titre, et la revue Sans Frontières, pourraient être liés à l'un des dédicataires présents dans la version autographe de la dédicace conservée dans les archives, mais dont le nom a disparu de la version publiée. Je reproduis ci-dessous la dédicace autographe en repartant de sa traduction française et ajoute, en italique, la mention du dédicataire absent :

L'auteur remercie les correspondants anonymes de Libération pour avoir involontairement participé à son livre ; [ainsi que la journaliste algérienne Leïla Sebbar pour le titre de ses excellents reportages sur l'immigration nord-africaine en France] ; son prétendu homonyme, le lointain et invisible écrivain «Juan Goytisolo », pour la reproduction de ses élucubrations scientifiques publiées dans le journal El País; et enfin le DAAD de Berlin pour la bourse qui lui a permis de terminer son roman [à] Kreuzberg dans l'atmosphère stimulante et paisible dont il avait besoin ( $\mathrm{PaB}$, p. 9 [et inédit MSI 3]).

Nul doute possible, on le voit : c'est pour le titre de ses reportages sur l'immigration nord-africaine que l'auteur avait pensé remercier Leïla Sebbar ${ }^{21}$. Or, il faut savoir que
Sans Frontières non seulement porte comme sous-titre Revue de l'Immigration et du Tiers-Monde, mais aussi que la revue a été créée au début des années quatre-vingt et que Leilla Sebbar y a tenu plusieurs années durant une rubrique intitulée « Mémoire »- où elle se faisait porte-parole des mémoires immigrées, déchirées entre deux cultures. En 1984, soit deux ans après la parution de Paysages après la bataille, elle y publia deux entretiens, le premier avec le cinéaste vietnamien Lam Lê, le second avec Juan Goytisolo, tous deux réunis sous le sous-titre «Chronique de l'exil » (Sebbar, 1984). Avant cela, et dans le prolongement de son travail de terrain, elle publia dans Les Temps modernes, en décembre 1980, puis en janvier 1981, deux livraisons d'un long article intitulé " Géographie de l'exil ». Celui-ci s'ouvrait et se fermait sur les mots que nous reproduisons ci-dessous - et on nous pardonnera la longueur des citations, mais elles ont l'avantage de cerner l'un des axes thématiques essentiels du roman de Juan Goytisolo, qui par bien des côtés est lui aussi chronique et géographie de l'exil.

«Le métro, c'est l'espace souterrain de Paris qui donne de l'exil la carte la plus lisible », écrit Sebbar (1980, p. 945) au début de la première livraison de son article, ce qui nous renvoie presque instantanément à la couverture de l'édition espagnole de Paysages après la bataille, dont les rabats reproduisaient un plan de métro de Paris, et aux propos du narrateur qui, anticipant de peu les récits de Marc Augé, Un ethnologue dans le métro (1986) et Le Métro revisité (2008)22, déclarait : «Examiner le plan du métro, c'est accéder au souvenir, au délire, à l'évasion. C'est parcourir

21. En mai 2009 et en janvier 2012, je me suis rendue à Marrakech pour rencontrer Juan Goytisolo et échanger à bâtons rompus avec lui sur sa manière de travailler. Juan Goytisolo n'a pas pu m'éclairer sur le pourquoi de la suppression des remerciements à Leïla Sebbar, car il n'en avait pas gardé le souvenir. Lors de ces entretiens, l'auteur m'a également montré les exemplaires des romans dans lesquels il se relit et apporte des corrections qui sont ensuite prises en compte par Antoni Munné, responsable de la réalisation des œuvres complètes en cours de publication à Barcelone (Galaxia Gutenberg/Círculo de Lectores).

22. Reprenant la perspective d'une « ethnologie du proche » qui fait la particularité des ouvrages de Marc Augé, et creusant ses observations sur le métro, Leïla Sebbar a récemment publié un ouvrage intitulé Métro. Instantanés (Paris, Éditions du Rocher, 2007), dans lequel le métro de Paris est l'occasion d'évoquer cette « Babel souterraine au-delà des mers, où se croisent les visages et les corps, les accents et les langues ». 
les monuments, abominations et horreurs de la capitale, les monuments, abominations et horreurs de son propre personnage, sans avoir à bouger de chez soi » $(\mathrm{PaB}$, p. 105).

Difficile aussi de ne pas lire les lignes ironiques du fragment goytisoléen "Symptômes de panique », où le lecteur est invité à « se rendre à l'évidence : l'Afrique commence sur les grands boulevards », à la lumière des lignes autobiographiques que traça Leilla Sebbar, en conclusion de la seconde livraison de son article, pour évoquer le trajet de métro qu'elle empruntait pour se rendre au journal Sans Frontières.

Depuis des années, au grand désespoir de nos lecteurs, il se refuse à visiter les quartiers paisibles et imposants, à prédominance aborigène, sous le fallacieux prétexte qu'ils lui donnent de l'asthme et, s'il y est forcé, il se munit à l'avance de tout le nécessaire - passeport, devises, certificat international de vaccination - comme s'il allait à un safari congolais ou à une expédition scientifique au Groënland $(P a B$, p. 155).

Comme souvent les exilés, je marche beaucoup dans Paris mais jamais de l'autre côté de la Seine. Je marche côté Sud, les mains libres, sans papiers, parfois sans argent, comme si je sortais pour un tour de quartier ou sur un chemin de campagne, comme si par ailleurs, je vivais dans la clandestinité sans indices compromettants sur moi.

Quand je suis allée pour la première fois au local de Sans Frontières, sans préméditation, je me suis retrouvée dans le métro avec, sur les genoux, un grand sac de toile dans lequel j'avais mis des mandarines, des oranges, des tartines beurrées. $\mathrm{J}$ 'avais pris soin de ranger mon passeport dans une poche avant, mon porte-monnaie et mes chèques dans l'autre. Un foulard à grandes fleurs je ne sais pourquoi, un livre et des journaux. J'allais en déplacement dans un pays lointain ; il me fallait des provisions de bouche et des magazines comme lorsqu'on prend le train... Je suis revenue le soir avec une tête de mouton grillée coupée en deux à cause de la cervelle... Les fois suivantes, j'ai oublié les provisions. Mais jamais le sac, avec je ne sais quoi dedans, qui me rattachait au Sud.

À mon premier retour de la Courneuve, dans le bus, j'ai demandé au chauffeur - au contrôleur - un ticket pour la France... (Sebbar, 1981, p. 1268-1269).

Point besoin de ces découvertes génétiques, il est vrai, pour pouvoir lire Paysages après la bataille comme possible «métaphore de l'immigration» (Kunz, 2003, p. 19). Car le roman est cartographie, topographie, géographie de l'exil. Et c'était bien ce que rappelaient les dernières lignes de l'actuel avant-dernier - peut-être en son temps dernier - fragment, « LA VILLE DES MORTS », écrit à trois reprises en majuscules dans les notes de synthèse 23 et apparaissant dans les deux premiers cas comme dernier élément listé.

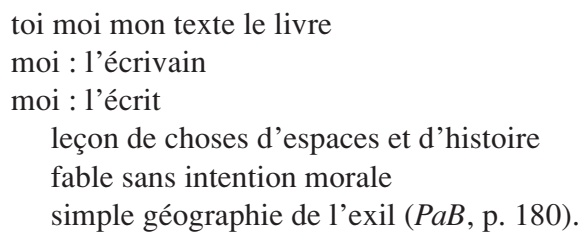

Ces lignes, plus encore la séquence dans sa totalité ont, c'est indéniable, vocation d'excipit, car elles condensent plusieurs objectifs et thèmes clés de ce « livre-ville » dans lequel nous, vous, il, je...

tu peux te promener dans les rues écrire t'égarer dans le double espace du livre et de la cité désorienter te désorienter : épandre la matière du récit dans la rose des vents au hasard des impondérables et des accidents : textes-aigrette à la merci d'une brise véhicule d'une pollinisation subtile ( $\mathrm{PaB}$, p. 179).

Enfin, cette hypothèse pourrait être corroborée par une note de régie écrite dans le coin supérieur droit de la feuille MSI 307, mise au net non définitive de « La ville des morts », car les substitutions y sont nombreuses si on la compare avec le texte publié. En effet, on peut y lire les mots suivants écrits pour partie en majuscules : « AÑADIR al final », c'est-à-dire « AJOUTER à la fin » (fig. 6).

\section{« AJOUTER à la fin »}

On ne peut exclure que tels auraient pu être les mots de la fin... si le hasard ne s'était pas chargé de venir semer un nouvel ordre, un nouveau désordre que le seul examen des brouillons n'aurait probablement pas suffi à éclairer.

Comme on le sait, Paysages après la bataille se referme - et s'ouvre - sur « L'ordre des facteurs n'altère pas le

23. Voir, par exemple, la dernière entrée du feuillet MSII 207 reproduit ci-dessus (fig. 3). 


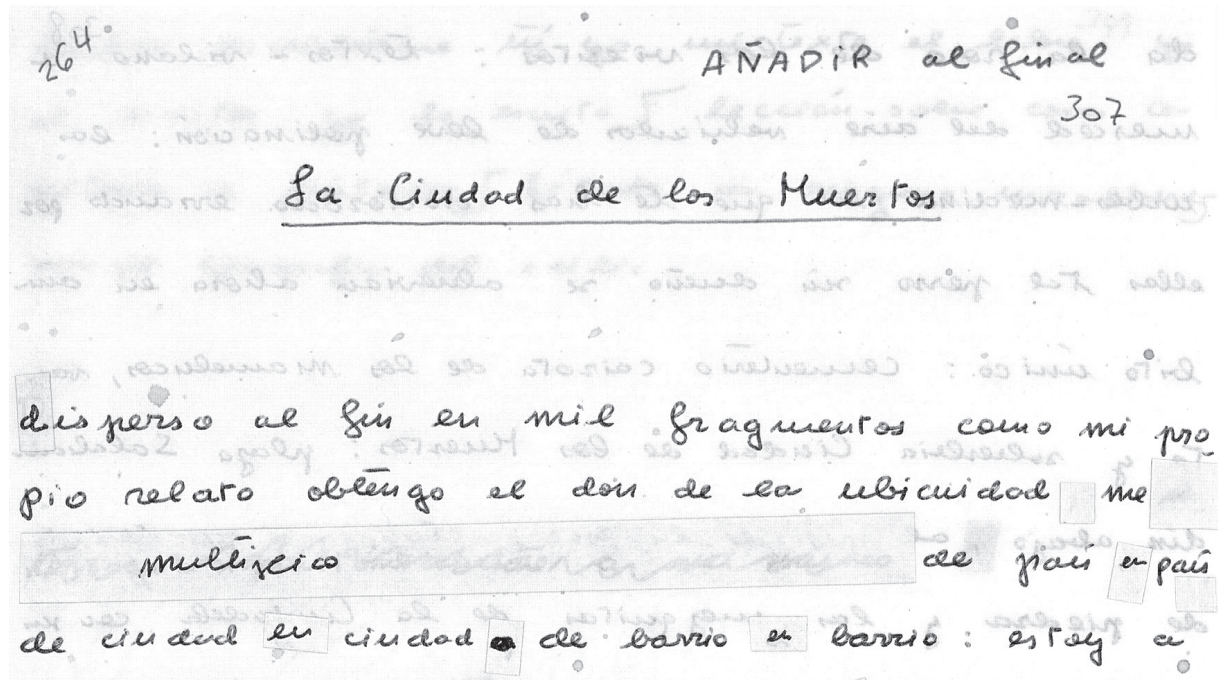

Fig. 6 : MSI 307 (@ Fonds Goytisolo)

produit », une séquence aux contours apparemment logiques ou mathématiques qui, au lieu de privilégier le seul axe historico-géographique de l'exil qui sous-tend les flâneries du protagoniste, nous renvoie, dans des jeux de miroir sans fin, à trois facettes de la personnalité du héros - présumé pédophile, chroniqueur scientifique et activiste engagé -, trois facettes qui réfractent à leur tour les trois types d'écrits mentionnés dans la dédicace que le «scribouillard » copie à longueur de roman - et on relèvera la présence des guillemets encadrant les trois attitudes, pour éviter toute lecture au premier degré, vu que celles-ci renvoient aussi au matériel collationné dans la presse.

Oublions tout ce qui est « syntagme verbal », « expérimentation », « niveaux de lecture », « intention ludique ». Disons simplement, comme les mathématiciens, que l'ordre des facteurs n'altère pas le produit.

Assis à sa table de travail, le regard perdu sur les toits mansardés, les antennes de télévision, les cheminées, la coupole vert amande de l'Opéra, le ciel anémique et décoloré, notre héros pourrait écrire :

« Cherche petite fille impubère candidement perverse pour me donner de temps en temps la fessée, pour me poudrer le derrière et changer mes langes quand ils sont mouillés. » ou

«Soyez prévoyants, agressions et désastres radioactifs vous menacent, protégez-vous, protégez votre famille grâce à notre modèle d'ombrelle nucléaire efficace et coquet. » ou encore

«Après plusieurs siècles d'un silence lâche et complice sur le génocide du peuple otèke exterminé par les hordes tartares avec la connivence de l'Empire céleste et d'autres puissances asiatiques, nous avons décidé de passer à l'action » $(\mathrm{PaB}$, p. 180-181).
Tout comme dans le cas de « La ville des morts », il existe des traces génétiques liées aux origines de cette séquence tardive qui s'est vue attribuer un titre initialement inscrit dans l'en-tête du brouillon de la séquence [74] qui prendra alors le titre du roman, sans qu'il en reste de trace dans les brouillons. Ce changement est particulièrement intéressant car les toutes premières lignes de la séquence $-\mathrm{y}$ compris les mots du titre «El orden de los factores no altera el producto » - ont été conservées à côté de la manchette d'un numéro du Monde, qui peut être daté avec une grande précision grâce à la présence d'éléments sémantiques marqués qui peuvent être déchiffrés mais n'avaient pas vocation à être lus (MSI 323). Il en va ainsi des mots « [attenta]t de la rue Marbeuf », «"Carlos" ou les services syriens » (fig. 7). Ces informations renvoient à l'attentat à la voiture piégée qui explosa le 22 avril 1982, devant les bureaux de la revue Al-Watan al-Arabi, rue Marbeuf, à Paris. Cet attentat non revendiqué fut attribué au terroriste vénézuélien Ilitch Ramírez Sánchez alias Carlos. L'élément pertinent ici est la date, car les notes autographes sur la coupure du Monde impliquent que cette séquence - dont il existe deux états n'a pas été rédigée avant le 22 avril 1982, c'est-à-dire six mois à peine avant la sortie du roman, paru en novembre. Enfin, et je terminerai là-dessus, cette conjonction de facteurs prend un sens particulièrement symbolique à la lumière d'une récente anecdote que Juan Goytisolo a racontée dans le prologue du volume des Euvres complètes dans lequel est inclus le roman. Il y déclara se souvenir des origines exactes du titre donné à cette séquence. Origine insolite, car fruit véritable du hasard et d'un gallicisme bien involontaire de sa concierge. 


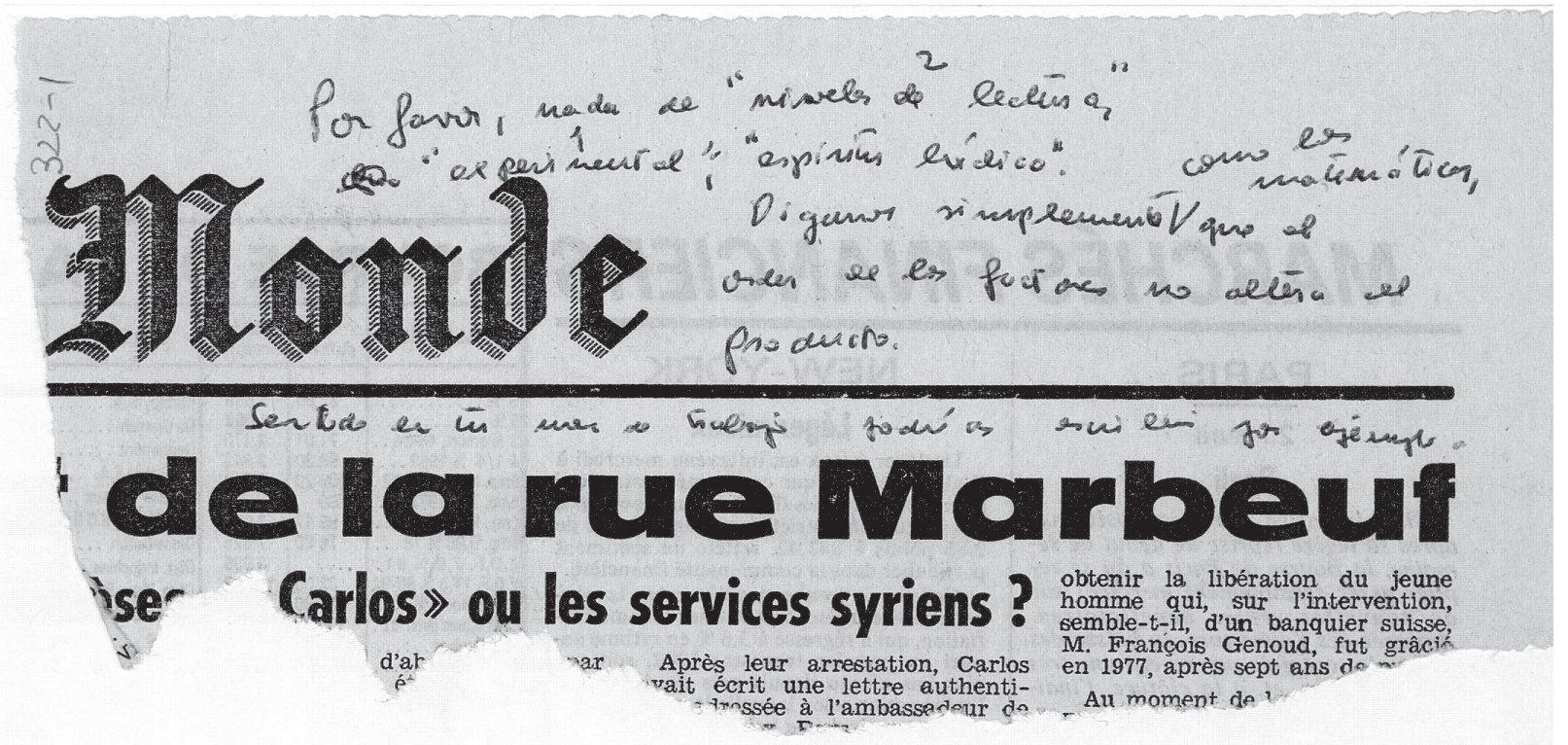

Fig. 7 : MSI 323 (@ Fonds Goytisolo)

\begin{abstract}
Alors que j'écrivais la dernière séquence, le casse-tête une fois terminé, je ne parvenais pas à lui trouver un titre et j'écartais les uns après les autres tous ceux qui me passaient par la tête. Dans cet embarras, le hasard vint à mon secours. J'attendais une lettre par service express, mais la concierge espagnole de l'immeuble de la rue Poissonnière m'apporta seulement le courrier ordinaire. Une heure après, elle frappa à la porte pour me remettre la lettre qui aurait normalement dû arriver par courrier prioritaire. Dans un savoureux mélange d'espagnol et de français, elle m'expliqua : «Los factores (facteurs, carteros) han venido en orden distinto » [Les facteurs sont venus dans un ordre différent]. Je saisis sa phrase au vol et je notai : «L'ordre des facteurs n'altère pas le produit ». Sans qu'elle le sache, sa phrase avait mis le point final à mon livre (Goytisolo, 2006, III, p. 40 [je traduis]).
\end{abstract}

Il m'est très difficile de remettre en question, plus encore en doute, le souvenir de l'auteur. Toutefois, les éléments du dossier génétique que je viens d'évoquer : présence du titre entre crochets au-dessus d'une autre séquence, ce qui laisse penser à un déplacement potentiel ; substitution d'un possible titre initial (« Géographie de l'exil ») et antéposition d'une séquence pensée comme fin (« La ville des morts ») ; disparition de l'un des dédicataires ; enfin, coupure du Monde datée du mois d'avril 1982 et, par conséquent, date très tardive de rédaction de cette séquence par rapport à la date de publication du roman; tous ces éléments laissent penser au mieux à la rédaction de toute la séquence, et non du seul titre, sous le coup du hasard, au pire aux possibles pièges qui guettent la mémoire, qu'elle soit autobiographique ou autocritique. «Impossible d'échapper au dilemme : reconstruire le passé sera toujours une manière sûre de le trahir, dans la mesure où on lui confère une cohérence a posteriori, où on le façonne pour lui donner une continuité artistique », écrit Juan Goytisolo à la fin de son autobiographie (1988, p. 349).

Nul doute non plus - et telle serait, à mon sens, la leçon qui se dégage de cette première étude génétique exhaustive menée à bien sur un corpus hispanique - que ce piège et cette explication, qui renvoient doublement à l'illusion téléologique, c'est-à-dire, d'une part, d'un «telos [biographique] avec ce qu'il implique de linéarité sous-jacente » (Dosse, 2005, p. 228), d'autre part, d'un telos scripturaire qui renvoie à un «idéal de finalité, d'efficacité et de perfection » (Grésillon, 1994, p. 137), peuvent être réinterrogés à la lumière de la genèse.

Dans sa très belle étude sur Un coeur simple de Flaubert, Debray Genette a ainsi montré pourquoi excipit et incipit 
ne sont pas finalement deux lieux stratégiques qui se renvoient en miroir.

Tout aléatoire qu'il peut être, un incipit n'en conserve pas moins son caractère primordial [...] et décisif. Or on pourrait, à la limite, renverser ce paradoxe à propos de l'excipit [sic] : tout programmé qu'il semble devoir être par le début et le cours du récit, il n'en conserve pas moins son caractère arbitraire, au point qu'il peut parfois disparaître, de gré ou de force, sans entamer la réputation et l'efficacité d'un récit (Debray Genette, 1988, p. 85-86).

D'un point de vue herméneutique, l'ajout de la séquence «L'ordre des facteurs n'altère pas le produit » a doté l'œuvre d'une circularité - les mots de la fin nous renvoyant à la «monstrueuse "Hécatombe" initiale »- absente de «La ville des morts » qui se refermait sur la dislocation, sur l'émiettement, sur la dispersion du récit et de la personnalité. D'un point de vue génétique, l'ajout de la séquence remet l'accent sur la facette du scribe, tout en illustrant le caractère processuel, le perpetuum mobile de l'écriture ; d'une écriture faite de ratures, d'ajouts, de déplacements, de substitutions, en bref, de réécritures successives que seule la mort de l'auteur, et non pas même l'édition ou la réédition, vient interrompre. «Une œuvre littéraire s'élabore au cours de différentes phases : elle se corrige, se réécrit, s'épure non seulement pendant la composition, mais aussi quand elle est déjà imprimée », est venu très judicieusement nous rappeler Juan Goytisolo dans le prologue au premier volume des Euvres complètes, relues, corrigées et amendées (2005, p. 9-10 [je traduis ${ }^{24}$ ]).

\section{Références bibliographiques}

Augé Marc, 1986, Un ethnologue dans le métro, Paris, Hachette, coll. « Pluriel».

— 2008, Le Métro revisité, Paris, Éditions du Seuil, coll. « La librairie du XXIe siècle ».

BIASI Pierre-Marc de, 1998, «Qu'est-ce qu'un brouillon? Le cas Flaubert », dans Michel Contat et Daniel Ferrer (dir.), Pourquoi la critique génétique? Méthodes et théories, Paris, CNRS Éditions, p. 31-60.

— 2000, La Génétique des textes, Paris, Nathan, coll. « 128 ».

Debray Genette Raymonde, 1988, Métamorphoses du récit. Autour de Flaubert, Paris, Éditions du Seuil.
DeHENNIN Elsa, 1996, « Mutaciones discursivas en la obra de Juan Goytisolo », Del realismo español al fantástico hispanoamericano. Estudios de narratología, Genève, Droz, p. 135-173.

Dosse François, 2005, Le Pari biographique. Écrire une vie, Paris, La Découverte.

Goytisolo Juan, 1982, Paisajes después de la batalla, México, Montesinos.

- 1982, Crónicas sarracinas, Barcelona, Ibérica de Ediciones.

- 1985, Paysages après la bataille, trad. Aline Schulman, Paris [Fayard, 1985], Stock, 1992.

- 1985, Contracorrientes, Barcelona, Montesinos.

- 1985, Los reinos de Taifa, Barcelona, Seix Barral.

— 1988, Les Royaumes déchirés, trad. Joëlle Lacor, Paris [Fayard, 1988], Stock, 1995.

— 2000, « Bouvard et Pécuchet sur les pas de Cervantès », Les Cervantiades, trad. Abdellatif Ben Salem avec la collaboration de l'auteur, Paris, BnF, 2000, p. 37-46 [conférence prononcée le 28 janvier 1998 dans l'auditorium de la BnF].

- 2005, Obras completas I. Novelas y ensayo (1954-1959), Barcelona, Galaxia Gutenberg/Círculo de Lectores.

- 2006, Obras completas III. Novelas (1966-1982), Barcelona, Galaxia Gutenberg/Círculo de Lectores.

- 2007, Obras completas V. Autobiografía y viajes al mundo islámico, Barcelona, Galaxia Gutenberg/Círculo de Lectores.

- 2009, Obras completas VI. Ensayos literarios (1967-1999), Barcelona, Galaxia Gutenberg/Círculo de Lectores.

GrÉSILlon Almuth, 1994, Éléments de critique génétique. Lire les manuscrits modernes, Paris, PUF.

Kunz Marco, 2003, Juan Goytisolo: Metáforas de la migración, Madrid, Verbum.

Sebbar Leïla, 1980, « Géographie de l'exil (I) », Les Temps modernes, $\mathrm{n}^{\circ} 413$ (décembre), p. 945-972.

- 1981, « Géographie de 1'exil (II) », Les Temps modernes, $\mathrm{n}^{\circ} 414$ (janvier), p. 1233-1269.

— 1984, « Chronique de l'exil. Juan Goytisolo : le promeneur », Sans Frontières, $\mathrm{n}^{\circ} 88-89$, p. 52-53.

- 2007, Métro. Instantanés, Paris, Éditions du Rocher.

VAuthier Bénédicte, 2012a, «Genética de los manuscritos y genética textual. El caso de Paisajes después de la batalla de Juan Goytisolo », dans Crítica genética y edición de manuscritos hispánicos contemporáneos. Aportaciones a " una poética de transición entre estados », dir. B. Vauthier et J. Gamba Corradine, Salamanca, Ediciones Universidad Salamanca, p. 235-257.

Vauthier Bénédicte (éd.), 2012b, Juan Goytisolo, Paisajes después de la batalla, Salamanca, Ediciones Universidad Salamanca.

24. Voir supra, note 6. 
BÉNÉDicte VAUThier, professeure ordinaire de Littérature espagnole contemporaine à l'Université de Berne (Suisse), a enseigné à Madrid, Cáceres, Bonn, Liège et Tours. Spécialiste de l'œuvre de Miguel de Unamuno et du Cercle « B.M.V. » (Bakhtine, Volochinov, Medvedev), elle a notamment coordonné les ouvrages collectifs : Crítica genética y edición de manuscritos hispánicos contemporáneos. Aportaciones a una "poética de transición entre estados » (2012) et Mikhaïl Bakhtine, Valentin Volochinov et Pavel Medvedev dans les contextes européen et russe (2008) et édité les œuvres de Miguel de Unamuno, Manual de quijotismo (inédito). Cómo se hace una novela. Epistolario de Miguel de Unamuno/Jean Cassou (2005) et Amor y pedagogía. Epistolario Miguel de Unamuno/Santiago Valentí Camp (2002). Elle vient de terminer une édition critique (et génétique) de Paisajes después de la batalla de Juan Goytisolo (2012). benedicte.vauthier@rom.unibe.ch

\section{Résumés « Géographie de l'exil » de Juan Goytisolo}

Au départ d'un examen du dossier génétique de Paisajes después de la batalla (1982) (Paysages après la bataille) composé de près de 860 feuilles de brouillons autographes, de coupures de presse et de quelques feuilles de notes, je montrerai le déplacement qui semble s'être opéré au cours des étapes d'écriture d'un titre devenu d'abord le « mot de la fin» du roman, écarté ensuite pour une nouvelle fin romanesque probablement trouvée tardivement, qui pourrait avoir entraîné dans son sillage l'effacement d'une dédicace et d'une référence intertextuelle, qui, d'explicites dans un état $X$, sont devenues implicites dans la version définitive et publiée de l'œuvre.

From the study of the genetic record of Paisajes después de la batalla (1982) (Landscapes after the Battle) consisting of almost 860 sheets of autograph rough drafts, press clippings and some notes, I deduce the shifting that seems to have taken place during the writing stages of the title. The title was first included in the last paragraph (explicit) which eventually was replaced by a new novel-like ending, probably found at the end of the writing process. This change could have led to the erasure of a dedication and an intertextual reference, both of which were explicit in stage $\mathrm{X}$ and became implicit in the final published version of the work.

Ausgehend von der Untersuchung des genetischen Dossiers von Paisajes después de la batalla (1982), das aus fast 860 handschriftlichen Skizzen, Zeitungsausschnitten und einigen Notizzetteln besteht, wird hier die Verschiebung eines Titels aufgezeigt, die sich im Verlauf der Etappen des Schreibprozesses vollzogen zu haben scheint. Der Titel wurde zuerst zum Schlusswort des Romans und hat sich dann zu einem neuen, vermutlich zu einem späteren Zeitpunkt gefundenen, romanhaften Ende ausgeweitet, was eine Widmung und eine intertextuelle Referenz ausgelöscht haben könnte, welche in einem Zustand X explizit waren und in der definitiven und veröffentlichten Version des Werks implizit geworden sind.
Partiendo del examen del dossier genético de Paisajes después de la batalla (1982), compuesto por cerca de 800 hojas de manuscritos autógrafos, recortes de prensa y algunos hojas de notas, estudio el desplazamiento que parece haberse realizado durante las etapas de escritura de un título, que primero se transforma en "palabras finales" de la novela, es reemplazado luego por otro final novelesco, probablemente ideado tardíamente, que podría haber arrastrado consigo la desaparición de una dedicatoria y de una referencia intertextual que, siendo explícitas en un estado X, quedaron implícitas en la versión definitiva y publicada de la obra.

A partir do exame do dossier genético de Paisajes después de la batalla (1982), composto por cerca de 860 folhas de rascunhos autógrafos, recortes de imprensa e algumas folhas de apontamentos, mostrarei a deslocação do título, que parece ter-se operado durante as etapas de escrita. Inicialmente, o título tinha a última palavra da novela, mas foi seguidamente afastado por um novo remate romanesco, encontrado tardiamente. De caminho, esbateram-se uma dedicatória e uma referência intertextual, ambas explícitas num estado X, mas tornadas implícitas na versão definitiva e publicada da obra.

Dall'analisi genetica del dossier di Paisajes después de la batalla (1982), composto da circa 860 fogli autografi, da ritagli di stampa e da annotazioni, mostrerò il passaggio che sembra essersi operato nel corso delle tappe di scrittura del titolo: dapprima incluso nel finale del romanzo, fu poi scartato per un nuovo finale, probabilmente trovato più tardi. Questa modifica potrebbe avere condotto alla cancellazione di una dedica e di un riferimento intertestuale che, espliciti ad un certo punto, divennero impliciti nella versione finale e pubblicata dell'opera. 